\title{
Depressive Symptoms and Sleep Disturbances in Korean American Women
}

Asian/Pacific Island Nursing Journal

Volume 1(4): 183-193

(C)Author(s) 2016

http://digitalscholarship.unlv.edu/apin/

\author{
Eunjung Kima, Sinhye Kimb ${ }^{\mathrm{b}}$, and Kevin C. Cain ${ }^{\mathrm{a}}$
}

\begin{abstract}
The purpose of this study was to examine the relationship between depressive symptoms and sleep disturbances among Korean American women. Forty-nine women completed the Center for Epidemiologic Studies Depression Scale, the Pittsburgh Sleep Quality Index, and revised Acculturation Rating Scale for Mexican Americans-II. Overall, participants scored $12.56(S D=9.93)$ on the Center for Epidemiologic Studies Depression Scale, $5.31(S D=3.01)$ on the Pittsburgh Sleep Quality Index, and -2.27 $(S D=1.64)$ on the Acculturation Rating Scale for Mexican Americans-II. Approximately $29 \%$ of the women $(n=14)$ scored 16 or higher on the Center for Epidemiologic Studies Depression Scale indicating that they had elevated depressive symptoms, and 39\% $(n=19)$ scored 6 or higher on the Pittsburgh Sleep Quality Index, which indicated that they had sleep disturbances. Results from the stepwise multiple regression, controlling for the degree of the women's acculturation, indicated that sleep disturbances $(\beta=.39, p=.004)$ were significantly positively related to depressive symptoms, $F(2,46)=7.27, p$ $=.002$ and the model explained $24 \%$ of the variance in women's depressive symptoms. When taking care of Korean American women who have elevated depressive symptoms, their sleep disturbances need to be assessed. Health practitioners need to assess for depressive symptoms in women with sleep disturbances.
\end{abstract}

Keywords: Korean American women, sleep disturbances, depressive symptoms, acculturation

Depression is a global health problem with evidence of racial/ethnic inequities (U.S. Department of Health and Human Services, 2001). For example, in a national study with mothers of young children living in the United States (U.S.), 44.4\% Korean American (KA) mothers, compared to $38.5 \%$ of nonHispanic White American mothers, reported depressive symptoms (Huang, Wong, Ronzio, \& Yu, 2007). In this paper, the term depressive symptoms is used to indicate symptoms of depression such as feeling sad, lonely, and helpless measured using screening tools (e.g., Center for Epidemiologic Studies Depression Scale, CES-D $\geq 16$ ), rather than depression, which is usually assessed using diagnostic criteria (i.e., DSM-V). Studies have established that sleep disturbances are major factors for depression (Baglioni et al., 2011; Paunio et al., 2015). Mothers of young children are particularly vulnerable to having sleep disturbances because their sleep is influenced by their children's sleep (Stranges, Tigbe, Gomez-Olive, Thorogood, \& Kandala, 2012). Further, depression was a risk factor for sleep disturbances (Franzen \& Buysse, 2008; Meerlo, Mistlberger, Jacobs, Heller, \& McGinty, 2009). Epidemiologic studies have demonstrated among people with diagnosed depression, up to $90 \%$ had sleep disturbances (Tsuno, Besset, \& Ritchie, 2005). A systematic review of 14 studies identified significant improvement in sleep disturbances after psychological treatment of depression (Yon et al., 2014). However, whether there is a link between depressive symptoms and sleep disturbances among KA women is not known. The purpose of this study was to examine the link between depressive symptoms and sleep disturbances among KA women.

${ }^{a}$ University of Washington, USA

${ }^{b}$ University of North Carolina, USA

Corresponding Author:

Eunjung Kim, PhD, ARNP, Associate professor, Department of Family and Child Nursing University of Washington Box 357262 Seattle WA 98195 Phone: (206) 543-8246, FAX: (206) 543-6656

Email: eunjungk@uw.edu 


\section{Depressive Symptoms Among Korean American Women}

Depression affects about $6.7 \%$ of U.S. adults each year, and women experience depression 70\% more than men in their lifetime (National Institute of Mental Health, 2010). According to the World Health Organization, depression impacts general health more than chronic illnesses (e.g., diabetes, arthritis, and asthma), and is linked with disability among adults aged 18 years and older (Moussavi et al., 2007; Murray et al., 2013). Depression is diagnosed through symptoms such as change in appetite, depressed mood, lack of interest or pleasure, feeling guilty, sleep disturbances, and lack of energy or concentration (American Psychiatric Association, 2013).

The scale used most commonly in assessing KAs' depressive symptoms is the Center for Epidemiologic Studies Depression Scale (CES-D). Researchers found higher total CES-D scores among KAs $(M=14.37-17.87)$ than those of other Asian American populations $(M=6.93-9.72)$ or the general community U.S. population $(M=14.37-17.87$ vs. $M$ = 7.94-9.25; Choi, 1997; Henderson et al., 2005; Huang et al., 2007; Kuo, 1984; Min, Moon, \& Lubben, 2005; Oh, Koeske, \& Sales, 2002; Radloff, 1977). KAs have reported higher total CES-D scores than Koreans living in Korea $(M=10.57$; Cho, Nam, \& Suh, 1998). In addition, KAs have reported a higher incidence of depressive symptoms that are possibly coming from the challenges they have faced in the U.S., such as dealing with new cultural standards, living situations, language, and socioeconomic positions (Jang, Kim, \& Chiriboga, 2011; Nicklett \& Burgard, 2009; Takeuchi et al., 2007). More KA women, compared to KA men, report depressive symptoms, which may be linked to chronic illnesses, lack of social resources, and disadvantaged social status such as low education and unmarried status (Ayers et al., 2009; Jang, Kim, et al., 2011). Approximately 30-49\% of KA women have reported depressive symptoms (Kim, 2013; Kim $\&$ Rew, 1994; Oh et al., 2002).

Researchers have found links between acculturation and the CES-D and its positive and negative affect subscales. Acculturation is defined as psychological and behavioral changes coming from contacts between immigrants' native culture and mainstream host culture (Berry, 2006). KAs who have adopted more of the U.S. culture, compared with those who have adopted less of the U.S. culture, reported higher scores on the positive affect subscale of the CES-D, which may have contributed to lower total CES-D scores among highly-acculturated KA immigrants (Kim, Seo, \& Cain, 2010). In other words, as KAs acculturated into the U.S. mainstream culture they adopted the U.S.-based open expression of positive affect (Jang, Kim, \& Chiriboga, 2005; Kim et al., 2010).

\section{Sleep Disturbances and Depressive Symptoms}

Approximately 50-70 million U.S. adults report sleep disturbances, which are defined as individuals' perceived troubles in sleep that result in daytime dysfunctioning although individuals have suitable chances and environments for sleep (Institute of Medicine [US] Committee on Sleep Medicine and Research, 2006). Sleep disturbances are related to emotional well-being and lack of sleep (as little as 12 hours per night) or fragmented sleep is related to increased illness and death rates (Centers for Disease Control and Prevention [CDC], 2011). While recommended sleep per night for adults is 7-9 hours (National Sleep Foundation, 2015), 35.3\% of U.S. adults reported that they sleep less than 7 hours (CDC, 2011).

Sleep disturbances are common among individuals who experience depression, and are highly prevalent among women (Aritake et al., 2015; Chan et al., 2014; Mallampalli \& Carter, 2014). About $40 \%$ of people with sleep disturbances have a psychiatric disorder, predominantly depression (Roth, 2007). However, results from an Australian longitudinal study indicated that compared to women without sleep disturbances, women with sleep disturbances at baseline were twice as likely to develop depression over 20 years (Jackson, Sztendur, Diamond, Byles, \& Bruck, 2014). These findings indicated that although sleep disturbances are considered a symptom of depression, it may precede depression.

Few studies have examined sleep disturbances among Koreans. Korean women who live in Korea and who have sleep disturbances experienced depression 4.3 times higher than those women without sleep disturbances (Cho et al., 2013). Cho et al. (2013) found that 20\% of Korean women were depressed using the CES-D (Cho et al., 2013). Among older KA adults, 38.7-82.6\% had sleep disturbances (Jang, Shin, Cho, Kim, \& Chiriboga, 2011; Sok, 2008). Among older KA adults, sleep disturbances were positively related to depressive symptoms based on the total CES-D scores and the CES-D's Positive and Negative Affect scores (Jang, Shin, et al., 2011).

Not much is known about the links among depression, acculturation, and sleep disturbances. In a study with Mexican American women, higher acculturation (measured by exposure to the U.S. culture before age 18), lower income, and higher depressive symptoms were related to sleep disturbances, accounting for $40 \%$ of the variance (Heilemann, Choudhury, Kury, \& Lee, 2012). 
Women who migrated to the U.S. at age of 17 years and younger were more likely to report sleep disturbances than those who came as an adult (Heilemann et al., 2012). Among pregnant Mexican American women, both acculturation and altered sleep were linked to depressive symptoms (D'AnnaHernandez, Garcia, Coussons-Read, Laudenslager, \& Ross, 2016). A high level of acculturation was linked to a high level of feeling refreshed when awaken in the morning, which in turn was negatively related to depressive symptoms (D'Anna-Hernandez et al., 2016).

Although depressive symptoms and sleep disturbances have been identified as significant health issues that are related, studies on this relationship among KAs are lacking. In view of the high incidence of depressive symptoms among KA women and the increasing number of KAs, more studies on depressive symptoms and sleep disturbances are needed so that health care providers can give evidence-based care. The aims of this study were to determine (a) the link between depressive symptoms and sleep disturbances and in KA women, and (b) how sleep disturbances differ based on the level of depressive symptoms.

\section{Method}

\section{Design and Sample}

Using a correlational cross-sectional research design, data were gathered from 49 KA women. Inclusion criteria were (a) having a child aged 3-8 years old and (b) being a first generation immigrant born in Korea. Mothers of children under 8 years were chosen because results of previous studies indicated that interventions for children with behavior problems should target changing parenting practices of children up to age 8 (Bullis \& Walker, 1994; Webster-Stratton \& Hancock, 1998). There were no exclusion criteria. Power analysis showed $82 \%$ power to detect a difference between groups of .70 standard deviations $(d=1.0)$ with a sample size of 48. In this study, KA in refers to a person who was born in Korea, had ethnic Korean parents, and lived in the United States at the time of data collection.

\section{Instrumentation}

\section{Center for Epidemiologic Studies Depression Scale (CES-D)}

Depressive symptoms were measured using the CES-D (Radloff, 1977), which was developed for the general U.S. community population. The CES-D consists of 20 items that assess positive affect (e.g., happy), negative affect (e.g., sad), interpersonal difficulty (e.g., perceived unfriendliness), and somatic and retarded activity (e.g., poor appetite). In this paper, the sum score of depressive symptom items (i.e., negative affect, interpersonal difficulties, and somatic and retarded symptoms) are named as negative affect, whereas the sum score of positive affect items are named as positive affect. Respondents indicate the frequency of each item using a 4-point Likert-type scale from " 0 " (rarely) to " 3 " (almost or all of the time, 5-7 days/week). In calculating the total score, positive affect items were reverse-coded. The total CES-D score ranges between $0-60$. A score of 16 or higher indicates that the person is at risk for clinical depression (Radloff, 1977). Researchers found satisfactory reliability and content, construct, and concurrent validity for the Korean version of the CES-D (Kim, Han, \& Phillips, 2003; Noh, Avison, \& Kaspar, 1992; Oh et al., 2002).

\section{Pittsburgh Sleep Quality Index (PSQI)}

Women's perceived sleep disturbances were measured using the Pittsburgh Sleep Quality Index (Buysse, Reynolds, Monk, Berman, \& Kupfer, 1989). The PSQI is a 19-item instrument that assesses sleep disturbances in seven areas during the prior month: subjective sleep quality, sleep latency, sleep duration, habitual sleep efficiency, sleep disturbances, use of sleep medications, and daytime dysfunctioning. The 4-point Likert-type scale score ranges from " 0 " (no problem in that area) to " 3 " (the most problems in that area). The global PSQI scores range from 0-21 and scores 6 or higher indicate sleep disturbances. Cronbach's alpha for the American version was .83 (Paunio et al., 2015). Satisfactory reliability and validity were reported for the Korean version of the PSQI (Sohn, Kim, Lee, \& Cho, 2012).

\section{Modified Acculturation Rating Scale for Mexican Americans-II (ARSMA-II)}

A modified ARSMA-II was used to assess the level of acculturation. It is a 12-item, 5-point Likert-type scale instrument that assesses orientations to Mexican and American cultures such as language, ethnic interaction, and ethnic behaviors (Cuellar, Arnold, \& Maldonado, 1995). The word "Korean" was used instead of "Mexican" to fit the instrument with KA women. The modified subscales include American orientation (I enjoy speaking English) and Korean orientation (I enjoy speaking Korean). Scores range from 1-5 and the higher the score, the more culturally orientated the person is to that specified culture. By subtracting the Korean orientation score from the American orientation score, the women's acculturation scores were computed. Concurrent validity of the ARSMA-II was established by Cuellar et al. (1995). The Cronbach's alpha for the Mexican 
sample was .93 for American orientation and .88 for Mexican orientation.

\section{Procedure}

Self-report survey data were obtained as part of the Korean Parent Training Program that was delivered at six partnered Korean churches in the Pacific Northwest (Kim et al., 2014). The first author visited churches, explained the study, and distributed the survey that included both the Korean and English versions so that participants used a language they were comfortable with. Each participant completed the survey in their preferred place and time when the researcher was not present. Completed surveys were mailed to the researcher using the self-addressed stamped envelope that was provided to each participant. This study was approved by the researcher's Institutional Human Subjects Review Committee, and each participant signed the informed written consent form before participation. Each family that completed the survey received a ten-dollar gift certificate.

\section{Data Analysis}

Statistical Package for Social Science (SPSS) 20 was used to conduct the data analysis. Descriptive statistics such as distributions, means, standard deviations, frequencies, and ranges were computed. To answer the first study aim regarding the links between depressive symptoms and sleep disturbances in KA women, inter-correlations were examined among study variables. Then, stepwise multiple regression was used to predict depressive symptoms (dependent variables) from sleep disturbances (independent variable), controlling for acculturation. For the second aim regarding how sleep disturbances differ based on the level of depressive symptoms, the global PSQI and its subscale scores were calculated using the cut score of 16 in total CES-D; the mean differences were examined by $t$-test.

\section{Results}

\section{Descriptive Statistics of the Participants and Study Variables}

Table 1 presents the demographic characteristics of the study participants. Overall, KA women were in their 30s, received about 17 years of education, and lived in the U.S. roughly 11 years. Almost $41 \%$ had a family income of $\$ 80,000$ or higher. Six women used the English version questionnaires. Table 2 presents descriptive statistics of the study variables and their Cronbach's alpha score for the Korean version. As a group, the KA women had a mean total CES-D score of 12.56 (SD =
$9.93)$ and $29 \%$ of the sample $(n=14)$ scored 16 or higher. The mean score of the global PSQI was 5.31 $(S D=3.01)$ and $39 \%$ of the participants $(n=19)$ scored 6 or higher. The mean score of acculturation was $-2.27(S D=1.64)$, indicating the participants scored lower on American orientation than Korean orientation.

Table 1. Demographic Characteristics of Study Participants $(N=49)$

\begin{tabular}{ll}
\hline Variable & $\mathrm{M}(\mathrm{SD})$ or N $(\%)$ \\
\hline Age (years) & $36.39 \pm 4.07$ \\
Education (years) & $16.67 \pm 1.36$ \\
U.S. stay (years) & $11.25 \pm 8.63$ \\
Visa status & \\
$\quad$ Citizen & $23(46.9)$ \\
$\quad$ Permanent Resident & $15(30.6)$ \\
$\quad$ Temporary & $10(20.4)$ \\
$\quad$ Missing & $1(2.0)$ \\
Ethnic Identity & \\
$\quad$ Korean & $39(79.6)$ \\
$\quad$ Korean American & $9(18.4)$ \\
Work hours/weeks & $12.78 \pm 19.42$ \\
Children, number & $2.02 \pm 0.52$ \\
Child's age between 3-8 years & $5.48 \pm 1.55$ \\
Annual family income & \\
$\quad$ Under $\$ 40,000$ & $9(18.4)$ \\
$\quad$ \$40,001- $\$ 80,000$ & $17(34.7)$ \\
$\quad$ Over $\$ 80,001$ & $20(40.8)$ \\
\hline
\end{tabular}

\section{Pearson's Correlation Among Descriptive and Study Variables}

As depicted in Table 3, among demographic characteristics, none were significantly correlated with total CES-D, positive affect, and negative affect, except child age, which was negatively correlated with positive affect. The global PSQI scores were significantly negatively correlated with family income, women's work hours, and child age. When the correlation between demographic variables and PSQI subscales were examined, family income, the time lived in the U.S., work hours, and child age were significantly negatively correlated with various subscales. Finally, acculturation was positively correlated with education and time in the U.S.

Table 4 depicts the Pearson's correlations among the study variables. KA women's level of acculturation was significantly negatively correlated with total CES-D and negative affect while it was significantly positively correlated with positive affect. Thus, acculturation was controlled in further analyses. The results from the stepwise multiple regression, controlling for women's acculturation, indicated global PSQI scores $(\beta=.39, p=.004)$ were significantly positively related to depressive symptoms, $F(2,46)=7.27, p=.002$ as shown in Table 5. The model explained additional $15 \%$ of that variance in women's depressive symptoms. 
Table 2. Descriptive Statistics and Cronbach's Alpha on Study Variables $(N=49)$

\begin{tabular}{lll}
\hline Variable & $M \pm S D($ Range $)$ & Cronbach's Alpha \\
\hline Total CES-D & $12.56 \pm 9.93(0-48)$ & .90 \\
Positive affect & $8.13 \pm 2.67(3-12)$ & .86 \\
Negative affect & $8.51 \pm 8.20(0-39)$ & .91 \\
Global PSQI & $5.31 \pm 3.01(0-15)$ & .70 \\
$\quad$ Subjective sleep quality & $1.06 \pm 0.78(0-3)$ & \\
Sleep latency & $0.80 \pm 0.74(0-2)$ & \\
Sleep duration & $1.00 \pm 0.96(0-3)$ & \\
Habitual sleep efficiency & $0.37 \pm 0.81(0-3)$ & .92 \\
Sleep disturbance & $1.08 \pm 0.49(0-2)$ & .88 \\
Use of sleeping medication & $0.04 \pm 0.29(0-2)$ & \\
Daytime dysfunction & $0.96 \pm 0.79(0-3)$ & \\
Acculturation & $-2.27 \pm 1.64(-4.00-1.83)$ & \\
American orientation & $4.25 \pm 0.77(1.00-4.33)$ & \\
Korean orientation & $1.98 \pm 0.94(1.33-5.00)$ & \\
\hline
\end{tabular}

Table 3. Pearson's Correlation among Descriptive and Study Variables $(N=49)$

\begin{tabular}{lllllll}
\hline & Age & Education & Income & Time in U.S. & Work Hours & Child Age \\
\hline Total CES-D & -.02 & -.21 & -.12 & -.18 & -.23 & .05 \\
$\quad$ Positive affect & -.03 & .11 & -.12 & .21 & .10 & $-.32^{*}$ \\
$\quad$ Negative affect & -.03 & -.22 & -.19 & -.16 & -.24 & -.04 \\
Global PSQI & -.10 & -.12 & $-.35^{*}$ & -.20 & $-.32^{*}$ & $-.30^{*}$ \\
$\quad$ Subjective sleep quality & -.09 & -.22 & $-.31^{*}$ & -.13 & $-.42^{* *}$ & $-.40^{* *}$ \\
Sleep latency & -.18 & -.15 & $-.30^{*}$ & $-.31^{*}$ & $-.33^{*}$ & -.28 \\
Sleep duration & .12 & .06 & -.07 & .05 & -.20 & .06 \\
Habitual sleep efficiency & -.25 & -.25 & -.26 & -.03 & -.20 & -.21 \\
Sleep disturbance & -.19 & -.01 & -.28 & -.28 & -.16 & $-.46^{* * *}$ \\
Use of sleeping medication & .16 & .25 & -.24 & -.12 & .33 & .24 \\
Daytime dysfunction & .04 & -.02 & -.14 & -.18 & -.09 & -.15 \\
Acculturation & .11 & $.47 * * *$ & .22 & $.77 * *$ & .10 & .07 \\
\hline
\end{tabular}

Note. ${ }^{*} p<.05,{ }^{* *} p<.01,{ }^{* * *} p<.001$.

Table 4. Pearson's Correlation Among Study Variables $(N=49)$

\begin{tabular}{|c|c|c|c|c|c|c|c|c|c|c|c|c|}
\hline & CES-D & Pos & $\mathrm{Neg}$ & PSQI & Sub & Lat & Dur & Eff & Dist & Med & Day & Acc \\
\hline CES-D & -- & & & & & & & & & & & \\
\hline Pos & $.73 * * *$ & -- & & & & & & & & & & \\
\hline Neg & $.98 * * *$ & $-.57 * * *$ & -- & & & & & & & & & \\
\hline PSQI & $.42 * *$ & -.13 & $.48 * * *$ & -- & & & & & & & & \\
\hline Sub & $.35^{*}$ & -.13 & $.40 * *$ & $.82 * * *$ & -- & & & & & & & \\
\hline Lat & $.33 *$ & -.07 & $.37 * *$ & $.45^{* * * *}$ & $.35^{*}$ & -- & & & & & & \\
\hline Dur & .18 & -.08 & .20 & $.69 * * *$ & $.45^{* * * *}$ & -.03 & -- & & & & & \\
\hline Eff & .15 & .09 & .21 & $.72 * * *$ & $.50 * * *$ & .27 & $.54 * * *$ & -- & & & & \\
\hline Dist & .11 & .08 & .17 & $.55^{* * * *}$ & $.48 * * *$ & $.39 * *$ & .04 & .24 & -- & & & \\
\hline Med & .04 & -.18 & -.01 & .08 & -.01 & -.16 & .00 & -.07 & -.02 & -- & & \\
\hline Day & $.50 * * *$ & -.27 & $.53 * * *$ & $.65^{* * *}$ & $.48 * * *$ & .02 & $.41 * *$ & .19 & $.33^{*}$ & .19 & -- & \\
\hline Acc & $-.30 *$ & $.34 *$ & $-.29 *$ & -.13 & -.20 & -.19 & .17 & .09 & -.23 & -.07 & -.25 & -- \\
\hline
\end{tabular}

Note. Pos = Positive affect; Neg = Negative affect; Sub = Subjective sleep quality; Lat = Sleep latency; Dur = Sleep duration; Eff $=$ Habitual sleep efficiency; Dist $=$ Sleep disturbance; Med = Use of sleeping medication; Day = Daytime dysfunctioning; Acc = Acculturation; $* p<.05, * * p<.01, * * * p<.001$ (2-tailed). 
Asian/Pacific Island Nursing Journal, Vol. 1, Iss. 4 [2016], Art. 5

Table 5. Stepwise Multiple Regression Analysis Predicting Depressive Symptoms from Sleep Quality $(N=49)$

\begin{tabular}{llllll}
\hline \multicolumn{2}{l}{ Predictor Variables } & $\mathrm{B}(S E)$ & $\beta$ & $\Delta R^{2}$ & $F$ \\
\hline Step 1 & Acculturation & $-1.84(0.84)^{*}$ & $-.30^{*}$ & .09 & $4.73^{*}$ \\
Step 2 & Acculturation & $-1.53(0.79)$ & -.25 & .15 & $7.27^{* *}$ \\
& PSQI & $1.28(0.43)^{* *}$ & $.39^{* *}$ & & \\
\hline
\end{tabular}

Note. $* p<.05, * * p<.01$ (2-tailed).

\section{Korean American Women's Sleep Disturbances and Hours of Sleep per Depressive Status}

To examine KA women's sleep disturbances further and to address the second aim, the mean scores of the global PSQI and its subscale scores were calculated based on the total CES-D group using 16 as a cut point. As presented in Table 6, the mean score of the global PSQI among women with total CES-D <16 was $4.52(S D=2.29)$, while it was $6.67(S D=3.63)$ among women with a total CES-D $\geq$ 16 , indicating that women with higher depressive symptoms had a mean score on the global PSQI that was higher than the cut point. These mean scores were statistically significantly different. When two groups were compared based on PSQI subscales, participants who had elevated depressive symptoms had significantly higher scores on subjective sleep quality and daytime dysfunction. Overall, $22.4 \%$ of the participants slept less than 7 hours per night. When participants were examined using two groups based on the cut score of 16 on the CES-D, no significant difference in the hours of sleep between the two groups was found.

Table 6. Comparison of PSQI Score According to the CES-D Cut Point

\begin{tabular}{lccc}
\hline & \multicolumn{2}{c}{ Korean American women } & $t$ \\
\cline { 2 - 3 } & CES-D $<16$ & CES-D $\geq 16$ \\
$(n=31)$ & $(n=18)$ & $-2.55^{*}$ \\
Global PSQI & $4.52 \pm 2.29$ & $6.67 \pm 3.63^{*}$ & $-2.82^{* *}$ \\
Subjective sleep quality & $0.84 \pm 0.69$ & $1.44 \pm 0.78$ & -1.94 \\
Sleep latency & $0.65 \pm 0.71$ & $1.06 \pm 0.73$ & -.31 \\
Sleep duration & $0.97 \pm 0.84$ & $1.06 \pm 1.16$ & -1.64 \\
Habitual sleep efficiency & $0.23 \pm 0.50$ & $0.61 \pm 1.15$ & -.32 \\
Sleep disturbance & $1.06 \pm 0.51$ & $1.11 \pm 0.47$ & -1.32 \\
Use of sleeping medication & $0.00 \pm 0.00$ & $0.11 \pm 0.47$ & $-2.24^{*}$ \\
Daytime dysfunctioning & $0.77 \pm 0.67$ & $1.28 \pm 0.90^{*}$ & -1.38 \\
Sleep hours per night & $7.51 \pm 1.28$ & $8.11 \pm 1.73$ & \\
\hline
\end{tabular}

Note. ${ }^{*} p<.05,{ }^{* *} p<.01$ (2-tailed).

\section{Discussion}

This study examined the links between KA women's depressive symptoms and sleep disturbances. Overall, $29 \%$ of KA women had elevated depressive symptoms, which is in line with prior findings that $30-31 \%$ of KA women living in the Pacific Northwest reported elevated depressive symptoms (Kim, 2009; Kim et al., 2010). Women's level of acculturation was negatively linked to depressive symptoms. This finding is also in accordance with earlier findings that when KAs were more acculturated into the U.S. culture, they reported fewer depressive symptoms than those who were less acculturated into the U.S. culture (Jang et al., 2005; Kim et al., 2010). A new finding is that acculturation was positively linked to CES-D's positive affect while it was negatively associated with CES-D's negative affect, indicating that women with higher acculturation tend to report more positive affect and fewer depressive symptoms. In a previous study, acculturation was not related to CES-D's negative affect (Kim et al., 2010). This finding needs to be further examined using a larger sample.

Acculturation was not related to sleep disturbances and this finding is different from previous study findings among Mexican Americans, where acculturation was related to sleep disturbances (D'Anna-Hernandez et al., 2016; Heilemann et al., 2012). This difference may be connected with the use of different measurement tools and/or the different ethnic groups sampled in these studies. In the present study, acculturation was measured using a modified ARSMA-II, which evaluated orientation to American and Korean culture as bidimensional acculturation (Cuellar, Arnold, \& Maldonado, 1995). Meanwhile, Hielemann et al. (2012) used age immigrated to the U.S. as an indication of acculturation and D'Anna- 
Hernandez et al. (2016) used the Acculturation Scale for Hispanics (Marin, Sabogal, Marin, OteroSabogal, \& Perez-Stable, 1987), which assesses media, language use, and ethnic social relations as unidimensional acculturation. Measures used to assess sleep disturbances were also different; while previous studies used the General Sleep Disturbance Scale (Heilemann et al., 2012; Lee, 1992) and selfreport Sleep Diaries (D'Anna-Hernandez et al., 2016), the current study used the Pittsburgh Sleep Quality Index (Buysse et al., 1989). These different research instruments may have elicited slightly different aspects of sleep quality resulting in the different findings.

The overall mean score of the global PSQI was $5.31 \pm 3.01$; however, $39 \%$ of participants $(n=$ 19) experienced sleep disturbances. This score was slightly lower than the mean score of 5.89 found among Korean mothers in Korea in a cross-cultural study (Mindell, Sadeh, Kwon, \& Goh, 2013). Further, a higher proportion of the Korean mothers had scores that indicated poorer sleep (53\%; Mindell et al., 2013) than in this KA sample (39\%). The average amount of sleep time for this study sample was 7.7 hours, and this was also slightly longer than 7.3 hours found among Korean mothers living in Korea (Mindell et al., 2013). The mean global PSQI score among those who scored a total CES-D < 16 was $4.80 \pm 2.44$, which is similar to the mean score of the healthy control group of Koreans living in Korea (4.06 \pm 2.08; Sohn et al., 2012).

When sleep quality was examined according to demographic variables, women who worked fewer hours, had a low family income, and had a younger child were more likely to report more sleep disturbances. These findings are consistent with existing evidence. In previous studies, mothers who worked full time had better sleep (Mindell et al., 2013), whereas mothers who worked part-time or were unemployed had poorer sleep (Heilemann et al., 2012; Soltani et al., 2012). In addition, women with less family income had more sleep disturbances (Heilemann et al., 2012; Soltani et al., 2012) and women with young children tended to have more sleep disturbances (Mindell et al., 2013). When mothers had young children who woke up more often in the night, this would wake the mothers up, resulting in sleep disturbances on the mothers, which in turn is related to elevated depressive symptoms.

Results indicated that KA women's sleep disturbances were significantly positively related with depressive symptoms controlling for their acculturation levels. The finding that sleep disturbances were linked to depressive symptoms is congruent with former findings among Korean female workers in Korea (Cho et al., 2013), Korean elderly in Korea (Chan et al., 2014), and older KA adults (Jang, Shin, et al., 2011). Sleep disturbances are part of the diagnostic conditions for depression (American Psychiatric Association, 2013), which favors finding a relationship between depression and sleep disturbances. Polysomnographic sleep researchers pointed out that altered sleep characteristics (troubled rapid eye movement), sleep regulation, and reduction in slow wave sleep production, were related to depression (Palagini, Baglioni, Ciapparelli, Gemignani, \& Riemann, 2013). A systematic review of 205 studies found that between $50-90 \%$ of individuals with depression complained about sleep disturbances (Tsuno et al., 2005).

When participants were divided into two groups using the cut score of 16 from the CES-D, differences were found between two groups in the global PSQI and two of its subscales. Participants who had elevated depressive symptoms had significantly less subjective sleep quality and significantly more daytime dysfunction. This means that, compared to women who have fewer depressive symptoms, women with elevated depressive symptoms experienced poorer overall sleep quality and more difficulty in staying awake during driving, eating foods, or interacting in social activities, and had more problems with having enough attention to get things done. However, these results need careful interpretation. While the global PSQI scores differed, the differences were only in terms of subjective sleep quality and daytime dysfunction. In addition, there is a strong connection between daytime dysfunction and depression. The women with elevated depressive symptoms did not report sleep alteration in terms of sleep duration, habitual sleep efficiency, and sleep disturbance. Therefore, the difference may be on the perception of sleep rather than sleep per se.

\section{Limitations}

One of the limitations of this study was that sampling bias may have occurred because the sample size was small, participants were recruited from KA churches, and all participants had children. Therefore, the results can only be generalized to childbearing KA women and must be cautiously interpreted. Second, this study used self-report questionnaires, which can be influenced by various aspects of information processing. A false relationship between two self-reported surveys may happen because participants with depressive symptoms are inclined to give negative answers to measures (Duggal, Carlson, Sroufe, \& Egeland, 2001). Another limitation was that this study did not assess fragmented sleep. Previous studies indicated that fragmented sleep was related to fatigue (Tsuchiya et al., 2014) and depression (Goyal, Gay, \& Lee, 2009). 
Finally, KAs may have elevated depressive symptoms due to other factors, such as lack of social support (Kim, 2009), low quality of life (Kim \& Rew, 1994), high acculturation stress (Choi, 1997), chronic pain or chronic disease (Schlosser, Gage, Kohli, \& Soler, 2016), or postpartum depression (Park, Karmaus, \& Zhang, 2015). In addition, the data were collected during the winter season, which could increase seasonal depression (Sandman et al., 2016).

Nonetheless, the findings provided initial evidence that depressive symptoms were linked to sleep disturbances in KA women, controlling for acculturation. By controlling for acculturation levels, this study tried to minimize possible differences in responses to depression and sleep disturbances among U.S. citizens, permanent residents, and sojourners.

\section{Conclusion}

The results from this study revealed statistically significant positive relationships between depressive symptoms and sleep disturbances for KA women, controlling for their acculturation levels. The findings can be used by health care practitioners who provide care to KA women with depressive symptoms. Assessing not only depressive symptoms but also sleep disturbances would be import because of the significant correlation between these two variables. If women have sleep disturbances, teaching good sleep hygiene practices is important as a recent study found that poor sleep hygiene was related to sleep disturbances (Yazdi, Loukzadeh, Moghaddam, \& Jalilolghadr, 2016). Future research may include developing a KA community-wide mental health promotion program that incorporates ways to decrease depressive symptoms including sleep hygiene. Longitudinal studies that examine the relationship between depressive symptoms and sleep disturbances changes over time is also necessary. Considering the adverse effect of maternal depression on children's developmental outcomes, women's depressive symptoms must be assessed in children's and family mental health intake.

\section{Funding}

This study was supported by a grant awarded to E. Kim "Faith-Based Partnership Promoting Korean Parenting and Child Mental Health” R21 MD005932.

\section{References}

American Psychiatric Association. (2013). Diagnostic and statistical manual of mental disorders (5th ed.).
Washington DC: American Psychiatric Association.

Aritake, S., Asaoka, S., Kagimura, T., Shimura, A., Futenma, K., Komada, Y., \& Inoue, Y. (2015). Internet-based survey of factors associated with subjective feeling of insomnia, depression, and low health-related quality of life among Japanese adults with sleep difficulty. International Journal of Behavioral Medicine, 22, 233-238. doi:10.1007/s12529-014-9421-7

Ayers, J. W., Hofstetter, C. R., Usita, P., Irvin, V. L., Kang, S., \& Hovell, M. F. (2009). Sorting out the competing effects of acculturation, immigrant stress, and social support on depression: A report on Korean women in California. The Journal of Nervous and Mental Disease, 197, 742-747. doi:10.1097/NMD.0b013e3181b96e9e

Baglioni, C., Battagliese, G., Feige, B., Spiegelhalder, K., Nissen, C., Voderholzer, U., . . Riemann, D. (2011). Insomnia as a predictor of depression: A meta-analytic evaluation of longitudinal epidemiological studies. Journal of Affective Disorders, 135(1-3), 10-19. doi:10.1016/j.jad. 2011.01.011

Berry, J. W. (2006). Acculturation: A conceptual overview. In M. C. Bornstein \& L. R. Cote (Eds.), Acculturation and parent-child relationships: Measurement and development (pp. 13-30). Mahwah, NJ: Lawrence Erbaum Associates, Publishers.

Bullis, M., \& Walker, H. M. (1994). Comprehensive school based systems for troubled youth. Eugene, OR: University of Oregon, Center on Human Development.

Buysse, D. J., Reynolds, C. F., III, Monk, T. H., Berman, S. R., \& Kupfer, D. J. (1989). The Pittsburgh Sleep Quality Index: A new instrument for psychiatric practice and research. Psychiatry Research, 28, 193-213. doi:10.1016/0165-1781(89)90047-4

Centers for Disease Control and Prevention. (2011). Effect of short sleep duration on daily activitiesUnited States, 2005-2008. MMWR. Morbidity and Mortality Weekly Report, 4(60), 239-242.

Chan, J. W., Lam, S. P., Li, S. X., Yu, M. W., Chan, N. Y., Zhang, J., \& Wing, Y. K. (2014). Eveningness and insomnia: Independent risk factors of nonremission in major depressive disorder. Sleep, 37(5), 911-917. doi:10.5665/sleep.3658

Cho, H. S., Kim, Y. W., Park, H. W., Lee, K. H., Jeong, B. G., Kang, Y. S., \& Park, K. S. (2013). The relationship between depressive symptoms among female workers and job stress and sleep quality. Annals of Occupational and Environmental Medicine, 25, 12. doi:10.1186/ 2052-4374-25-12

Cho, M. J., Nam, J. J., \& Suh, G. H. (1998). Prevalance of symptoms of depression in a nationwide samples 
of Korean adults. Psychiatry Reearchs, 81, 341352. doi:10.1016/S0165-1781(98)00122-X

Choi, G. (1997). Acculturative stress, social support, and depression in Korean American families. Journal of Family Social Work, 2, 81-97. doi:10.1300/ J039v02n01_06

Cuellar, I., Arnold, B., \& Maldonado, R. (1995). Acculturation Rating Scale for Mexican Americans-II: A revision of the original ARSMA Scale. Hispanic Journal of Behavioral Sciences, 17, 275-304. doi:10.1177/07399863950173001

D'Anna-Hernandez, K. L., Garcia, E., Coussons-Read, M., Laudenslager, M. L., \& Ross, R. G. (2016). Sleep moderates and mediates the relationship between acculturation and depressive symptoms in pregnant Mexican-American women. Maternal and Child Health Journal, 20, 422-433. doi:10.1007/s10995-015-1840-9

Duggal, S., Carlson, E., Sroufe, L. A., \& Egeland, B. (2001). Depressive symptomatology in childhood and adolescence. Development and Psychopathology, 13, 143-164. Doi:10.1017/S0954579401001109

Franzen, P. L., \& Buysse, D. J. (2008). Sleep disturbances and depression: Risk relationships for subsequent depression and therapeutic implications. Dialogues in Clinical Neuroscience, 10, 473-481.

Goyal, D., Gay, C., \& Lee, K. (2009). Fragmented maternal sleep is more strongly correlated with depressive symptoms than infant temperament at three months postpartum. Archives of Women's Mental Health, 12, 229-237. doi:10.1007/s00737-0090070-9

Heilemann, M. V., Choudhury, S. M., Kury, F. S., \& Lee, K. A. (2012). Factors associated with sleep disturbance in women of Mexican descent. Journal of Advanced Nursing, 68, 2256-2266. doi:10.1111/j.1365-2648.2011.05918.x

Henderson, C., Diez Roux, A. V., Jacobs Jr, D. R., Kiefe, C. I., West, D., \& Willians, D. R. (2005). Neighbourhood characteristics, individual level socioeconomic factors, and depressive symptoms in young adults: The CARDIA study. Journal of Epidemiology and Community Health, 59, 322328. doi:10.1136/jech.2003.018846

Huang, Z. J., Wong, F. Y., Ronzio, C. R., \& Yu, S. M. (2007). Depressive symptomatology and mental health help-seeking patterns of U.S.- and foreignbron mothers. Maternal and Child Health Journal, 11, 257-267. doi:10.1007/s10995-0060168-x

Institute of Medicine (US) Committee on Sleep Medicine and Research. (2006). The National Academies Collection: Reports funded by National Institutes of Health. In H. R. Colten \& B. M. Altevogt (Eds.), Sleep disorders and sleep deprivation: An unmet public health problem. Washington, DC: National Academies Press (US).
Jackson, M. L., Sztendur, E. M., Diamond, N. T., Byles, J. E., \& Bruck, D. (2014). Sleep difficulties and the development of depression and anxiety: A longitudinal study of young Australian women. Archives of Women's Mental Health, 17, 189198. doi:10.1007/s00737-014-0417-8

Jang, Y., Kim, G., \& Chiriboga, D. (2005). Acculturation and manifestation of depressive symptoms among Korean-American older adults. Aging \& Mental Health, 9, 500-507. doi:10.1080/ 13607860500193021

Jang, Y., Kim, G., \& Chiriboga, D. A. (2011). Gender differences in depressive symptoms among older Korean American immigrants. Social Work in Public Health, 26, 96-109. doi:10.1080/ 10911350902987003

Jang, Y., Shin, J., Cho, S., Kim, G., \& Chiriboga, D. A. (2011). The interactive role of chronic medical conditions and sleep disturbance in predicting depressive symptoms among Korean American older adults. Aging \& Mental Health, 15, 198203. doi:10.1080/13607860903493366

Kim, E. (2009). Multidimensional acculturation attitudes and depressive symptoms in Korean Americans. Issues in Mental Health Nursing, 30, 98-103. doi:10.1080/01612840802597663

Kim, E. (2013). Korean American parental depressive symptoms and children's mental health: The mediating role of parental acceptance-rejection. Journal of Pediatric Nursing, 28, 37-47. doi:10.1016/j.pedn.2012.04.004

Kim, E., Cain, K., Boutain, D., Chun, J., Kim, S., \& Im, H. (2014). Pilot study of the Korean Parent Training Program using a partial group randomized experimental study. Journal of Child and Adolescent Psychiatric Nursing, 27, 121-131. doi:10.1111/jcap.12071

Kim, E., Seo, K., \& Cain, K. C. (2010). Bi-dimensional acculturation and cultural response set in CES-D among Korean immigrants. Issues in Mental Health Nursing, 31, 576-583. doi:10.3109/ 01612840.2010 .483566

Kim, M., Han, H., \& Phillips, L. (2003). Metric equivalence assessment in cross-cultural research: Using an example of the Center for Epidemiological Studies -- Depression Scale. Journal of Nursing Measurement, 11, 5-18. doi:10.1891/ jnum.11.1.5.52061

Kim, S., \& Rew, L. (1994). Ethnic identity, role integration, quality of life, and depression in Korean-American women. Archives of Psychiatric Nursing, 8, 348-356. doi:10.1016/0883-9417(94)90003-5

Kuo, W. H. (1984). Prevalence of depression among AsianAmericans. The Journal of Nervous and Mental Disease, 172, 449-457. doi:10.1097/00005053198408000-00002 
Lee, K. (1992). Self-reported sleep disturbances in employed women. Sleep, 15, 493-498.

Mallampalli, M. P., \& Carter, C. L. (2014). Exploring sex and gender differences in sleep health: A Society for Women's Health Research Report. Journal of Women's Health, 23, 553-562. doi:10.1089/ jwh.2014.4816

Marin, G., Sabogal, F., Marin, B. V., Otero-Sabogal, R., \& Perez-Stable, E. J. (1987). Development of a short acculturation scale for Hispanics. Hispanic Journal of Behavioral Sciences, 9, 183-205. doi:10.1177/07399863870092005

Meerlo, P., Mistlberger, R. E., Jacobs, B. L., Heller, H. C., \& McGinty, D. (2009). New neurons in the adult brain: The role of sleep and consequences of sleep loss. Sleep Medicine Reviews, 13, 187-194. doi:10.1016/j.smrv.2008.07.004

Min, J. W., Moon, A., \& Lubben, J. E. (2005). Determinants of psychological distress over time among older Korean immigrants and nonHispanic White elders: Evidence from a two wave panel study. Aging \& Mental Health, 9, 210-222. doi:10.1080/13607860500090011

Mindell, J. A., Sadeh, A., Kwon, R., \& Goh, D. Y. (2013). Cross-cultural comparison of maternal sleep. Sleep, 36, 1699-1706. doi:10.5665/sleep.3132

Moussavi, S., Chatterji, S., Verdes, E., Tandon, A., Patel, V., \& Ustun, B. (2007). Depression, chronic diseases, and decrements in health: Results from the World Health Surveys. Lancet, 370, 851-858. doi:10.1016/S0140-6736(07)61415-9

Murray, C. J., Atkinson, C., Bhalla, K., Birbeck, G., Burstein, R., Chou, D., . . . Wulf, S. (2013). The state of US health, 1990-2010: Burden of diseases, injuries, and risk factors. Journal of the American Medical Association, 310, 591-608. doi:10.1001/ jama.2013.13805

National Institute of Mental Health. (2010). What is depression. Retrieved from http://www.nimh.nih. gov/health/topics/depression/index.shtml

National Sleep Foundation. (2015). How much sleep do we really need? Retrieved from http://sleepfoundation.org/how-sleep-works/howmuch-sleep-do-we-really-need?page $=0 \% 2 \mathrm{C} 1$

Nicklett, E. J., \& Burgard, S. A. (2009). Downward social mobility and major depressive episodes among Latino and Asian-American immigrants to the United States. American Journal of Epidemiology, $\quad$ 170, 793-801. doi:10.1093/aje/kwp192

Noh, S., Avison, W. R., \& Kaspar, V. (1992). Depressive symptoms among Korean immigrants: Assessment of a translation of the Center for Epidemiologic Studies-Depression Scale. Psychological Assessment, 4, 84-91. doi:10.1037/ 1040-3590.4.1.84

Oh, Y., Koeske, G., \& Sales, E. (2002). Acculturation, stress, and depressive symptoms among Korean immigrants in the United States. The Journal of Social Psychology, 142, 511-526. doi:10.1080/ 00224540209603915

Palagini, L., Baglioni, C., Ciapparelli, A., Gemignani, A., \& Riemann, D. (2013). REM sleep dysregulation in depression: State of the art. Sleep Medicine Reviews, 17, 377-390. doi:10.1016/j.smrv.2012. 11.001

Park, J. H., Karmaus, W., \& Zhang, H. (2015). Prevalence of and Risk Factors for Depressive Symptoms in Korean Women throughout Pregnancy and in Postpartum Period. [Korean Soc Nurs Sci]. Asian Nursing Research, 9, 219-225. doi:10.1016/ j.anr.2015.03.004

Paunio, T., Korhonen, T., Hublin, C., Partinen, M., Koskenvuo, K., Koskenvuo, M., \& Kaprio, J. (2015). Poor sleep predicts symptoms of depression and disability retirement due to depression. Journal of Affective Disorders, 172, 381-389. doi:10.1016/j.jad.2014.10.002

Radloff, L. S. (1977). The CES-D Scale: A self-report depression scale for research in the general population. Applied Psychological Measurement, 1, 385-401. doi:10.1177/014662167700100306

Roth, T. (2007). Insomnia: Definition, prevalence, etiology, and consequences. Journal of Clinical Sleep Medicine, 3(5 Suppl), S7-10.

Sandman, N., Merikanto, I., Maattanen, H., Valli, K., Kronholm, E., Laatikainen, T., . . . Paunio, T. (2016). Winter is coming: Nightmares and sleep problems during seasonal affective disorder. Journal of Sleep Research, 25, 612-619. doi:10.1111/jsr.12416

Schlosser, R. J., Gage, S. E., Kohli, P., \& Soler, Z. M. (2016). Burden of illness: A systematic review of depression in chronic rhinosinusitis. Journal of Sleep Research, 30, 250-256. doi:10.2500/ ajra.2016.30.4343

Sohn, S., Kim, D., Lee, M., \& Cho, Y. W. (2012). The reliability and validity of the Korean version of the Pittsburg Sleep Quality Index. Sleep and Breathing, 16, 803-812. doi:10.1007/s11325-0110579-9

Sok, S. R. (2008). Sleep patterns and insomnia management in Korean-American older adult immigrants. Journal of Clinical Nursing, 17, 135-143. doi:10.1111/j.1365-2702.2006.01869.x

Soltani, M., Haytabakhsh, M. R., Najman, J. M., Williams, G. M., O'Callaghan, M. J., Bor, W., . . . Clavarino, A. (2012). Sleepless nights: The effect of socioeconomic status, physical activity, and lifestyle factors on sleep quality in a large cohort of Australian women. Archives of Women's Mental Health, 15, 237-247. doi:10.1007/s00737-012-0281-3

Stranges, S., Tigbe, W., Gomez-Olive, F. X., Thorogood, M., \& Kandala, N. B. (2012). Sleep problems: An emerging global epidemic? Findings from the 
INDEPTH WHO-SAGE study among more than 40,000 older adults from 8 countries across Africa and Asia. Sleep, 35, 1173-1181. doi:10.5665/ sleep.2012

Takeuchi, D., Zane, N., Hong, S., Chae, D., Gong, F., Gee, G. C., . . . Alegría, M. (2007). Immigrationrelated factors and mental disorders among Asian Americans. American Journal of Public Health, 97, 84-90. doi:10.2105/AJPH.2006.088401

Tsuchiya, M., Mori, E., Iwata, H., Sakajo, A., Maehara, K., Ozawa, H., . . . Saeki, A. (2014). Fragmented sleep and fatigue during postpartum hospitalization in older primiparous women. Nursing \& Health Sciences. doi:10.1111/nhs. 12157

Tsuno, N., Besset, A., \& Ritchie, K. (2005). Sleep and depression. The Journal of Clinical Psychiatry, 66, 1254-1269.

U.S. Department of Health and Human Services. (2001). Mental health: Culture, race, and ethnicity - A supplement to mental health: A report of the
Surgern General. Rockville, MD: Substance Abuse and Mental Health Services Administration (US). Retrieved from https://www.ncbi.nlm.nih.gov/books/NBK44243/

Webster-Stratton, C., \& Hancock, L. (1998). Training for parents of young children with conduct problems: Content, methods, and therapeutic processes. In C. E. Schaefer \& J. M. Briesmeister (Eds.), Handbook of parent training (pp. 98-152). New York, NY: John Wiley \& Sons.

Yazdi, Z., Loukzadeh, Z., Moghaddam, P., \& Jalilolghadr, S. (2016). Sleep Hygiene Practices and Their Relation to Sleep Quality in Medical Students of Qazvin University of Medical Sciences. Journal of Caring Sciences, 5, 153-160. doi:10.15171/ jcs.2016.016

Yon, A., Scogin, F., DiNapoli, E. A., McPherron, J., Arean, P. A., Bowman, D., . . Thompson, L. W. (2014). Do manualized treatments for depression reduce insomnia symptoms? Journal of Clinical Psychology, 70, 616-630. doi:10.1002/jclp.220 